Témoigner Témoigner. Entre histoire et mémoire

Getuigen Revue pluridisciplinaire de la Fondation Auschwitz

$124 \mid 2017$

La musique dans les camps

\title{
Présentation. La musique dans les camps
}

Presentation. Music in the camps

\section{Klaas Coulembier}

Traducteur : Carine Chauran

\section{(2) OpenEdition}

Journals

\section{Édition électronique}

URL : https://journals.openedition.org/temoigner/5730

DOI : 10.4000/temoigner.5730

ISSN : 2506-6390

Éditeur :

Éditions du Centre d'études et de documentation Mémoire d'Auschwitz, Éditions Kimé

Édition imprimée

Date de publication : 2 avril 2017

Pagination : $52-59$

ISBN : 978-2-930953-00-7

ISSN : 2031-4183

Référence électronique

Klaas Coulembier, «Présentation. La musique dans les camps », Témoigner. Entre histoire et mémoire [En ligne], 124 | 2017, mis en ligne le 30 novembre 2021, consulté le 01 décembre 2021. URL : http:// journals.openedition.org/temoigner/5730; DOI : https://doi.org/10.4000/temoigner.5730

Tous droits réservés 


\section{DOSSIER}

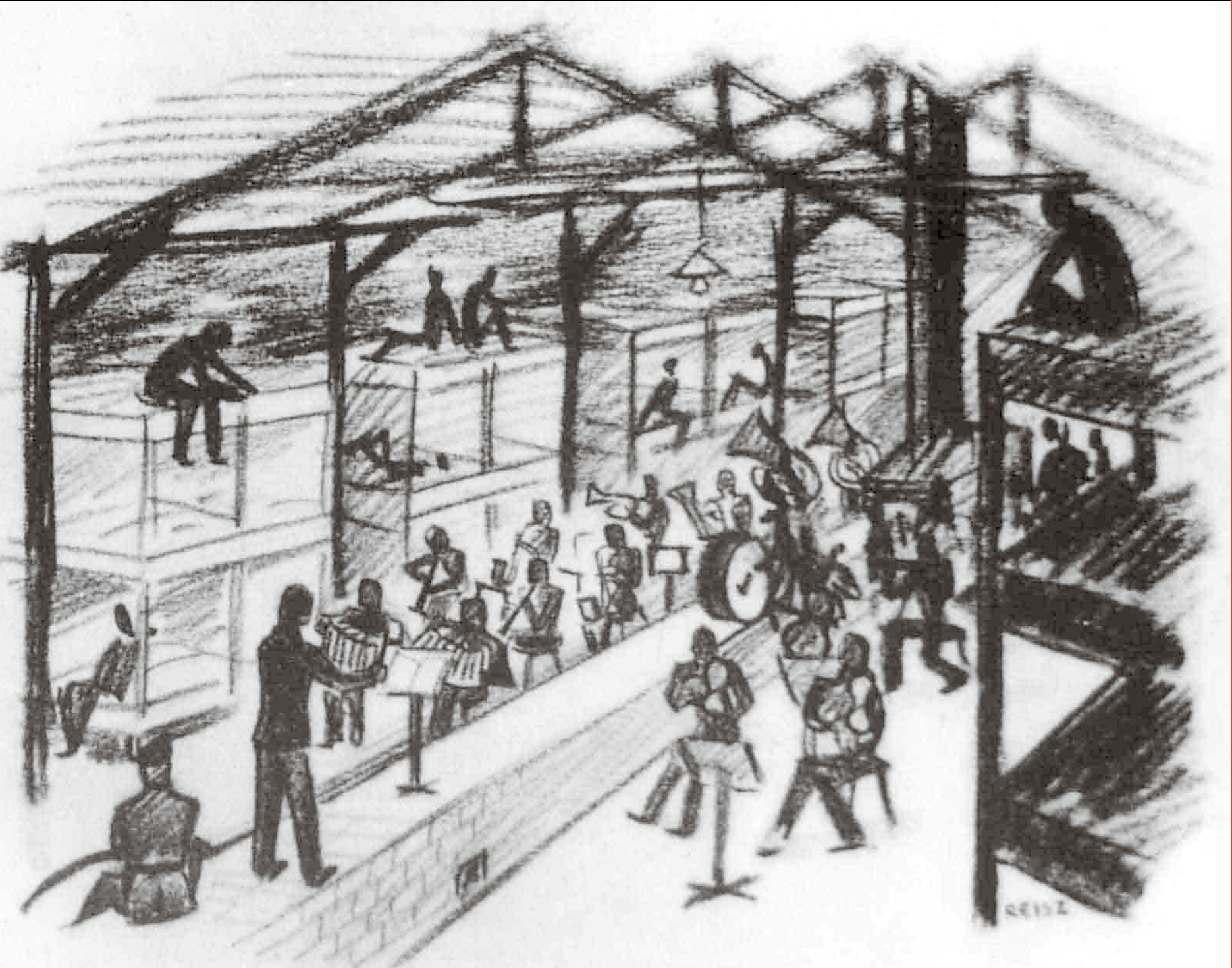

\section{LAMUSIQUE MUSIC DANS LES INTHE}

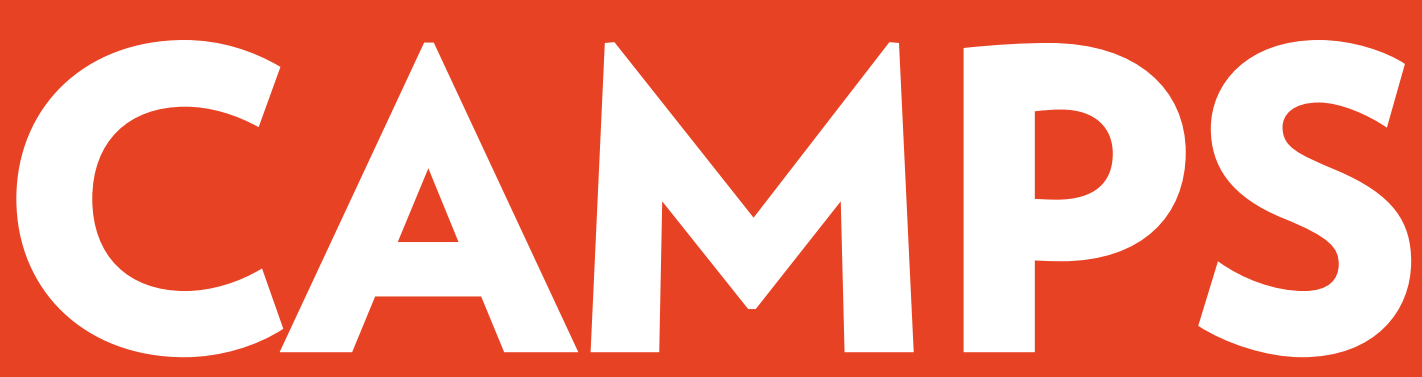

La musique a fait partie intégrante de lunivers concentrationnaire, nazi et autre. Quel genre de musique était composée et exécutée, et quel en était le rôle exact dans les camps? Facteur de survie et de résistance pour les détenus, une façon pour eux d'exprimer leur espérance et leur humanité - ou, au contraire, instrument d'oppression exploité par les bourreaux? Quelle est la fonction de la musique dans le travail de mémoire suivant lexpérience de violence et de souffrance extrêmes? Ce dossier propose de parcourir ces problématiques.

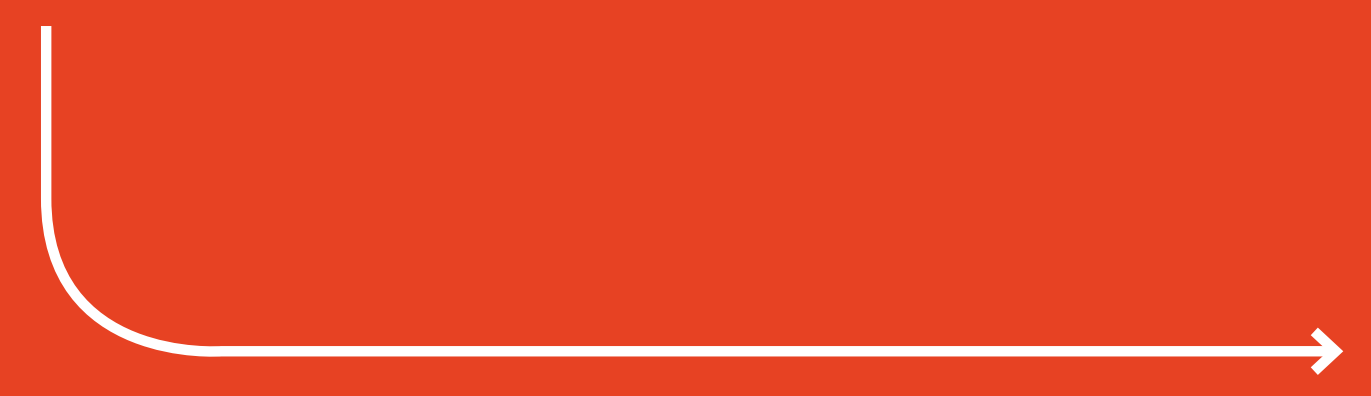


Un enfant dans le noir saisipar la peur, se rassure en chantonnant. Il marche, s'arrête au gréde sa chanson. Perdu, il s'abrite comme il peut, ou s'oriente tant bien que mal avec sa petite chanson. Celle-ciest comme l'esquisse d'un centre stable et calme, stabilisant et calmant, au sein du chaos. (Deleuze \& Guattari 1980, 382)

A child in the dark, gripped with fear, comforts himself by singing under his breath. He walks and halts to his song. Lost, he takes shelter, or orients himself with his little song as best he can. The song is like a rough sketch of a calming and stabilizing, calm and stable, center in the heart of chaos. (Deleuze \& Guattari 1987, 311)

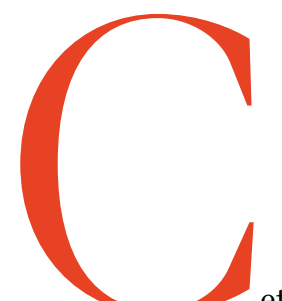
ouvrage philosophique Mille Plateaux de Gilles Deleuze et Félix Guattari introduit l'une des nombreuses fonctions de la musique. Dans les moments difficiles, quand nous avons peur, nous avon souvent recours, de façon consciente ou non, à la musique comme moyen de réconfort ou comme elément auque nous raccrocher. Deleuze et Guatta dépeignent la musique comme une forc interne contre toute crainte de l'extérieur. Dans l'angoisse, l'ignorance ou la peur, les personnes se tournent souven vers la musique conme renede, peutêtre même comme moyen de survie.

La musique a eu plusieurs fonctions dans les camps de concentration et les ghettos durant la Seconde Guerre mondiale et dans les années qui l'on précédée. En fait, l'essence de la création musicale semble tellementéloignée de l'horreur quotidienne des camps que toute musique dans un tel contexte constitue un élément révélateur. Une musique peut surgir de différentes tant fredonnement du prisonnier face à une mort certaine, jusquaux chants utilisés par l'éhontée propagande nazie. Le premier exemple trouve un écho dans a citation de Deleuze ci-dessus. Pour le deuxième, le plus flagrant est celui du ghetto de Theresienstadt - où furent et artistes - qui a vu le développemen d'une vie culturelle florissante, façade censeee « rassurer » la Croix-Rouge et donc le grand public. Entre ces deux extrêmes, la musique pouvait être utilisee comme divertissement, diversion, punition, torture, endoctrinement, etc.

La musique constitue l'un des nombreux fils par lesquels chacun peut raconter l'histoire de la Shoah et de histoire trace une perspective bien plus large que celle des seuls camps de concentration. Les contributions à ce façons, depuis le plus intime et réconforregroupes de nombreux intellectuel la Seconde Guerre mondiale, et cette dossier sont surtout axées sur la présence de la musique dans les camps, mais il est également intéressant d'observer le rôle qu’elle a joué dans l'idéologie nazie.

\section{La bonne musique et la mauvaise \\ musique}

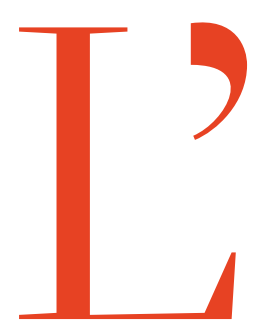
de base du processus, long et complexe, qui a permis les cruautés commises lor de la Shoah consiste en la distinctio sans ambiguité du bien et du mal, et l'exclusion de certains groupes de populations. Les Juifs n'ont pas etéles seule victimes de cette idéologie: les Tsiganes, les handicapes physiques ou les personnes souffrant de troubles mentaux en ontégalement subi les conséquence. Dans la société idéale aryenne, il ne pouvaity avoir de place que pour les productions artistiques qui illustraient, voire stimulaient, la culture pure et élevé du peuple allemand. Richard Wagne (1813-1883) était souvent cité comm exemple et ses textes antisemites (bien quècrits dans un contexte politique diférent de celui des annees 1930) ont été adoptés avec ferveur afin de souligner longue tradition de la suprématie culturelle allemande. Les compositions ou autres produits artistiques considere commeinappropriésétaient qualifiés de «dégénérés» (entartet). En 1937, plus de
600 œuvres ont été retirées des musées allemands car qualifiées comme telles. Plutôt que de détruire immédiatement ces peintures et ces sculptures, les autorités les ont réunies dans une exposition intitulée «Entartete Kunst», présentée dans les principales villes d'Allemagne et d'Autriche (Schuster, 1988). Cette exposition visait à montrer comment certains artistes avaient choisi le mauvais chemin. Selon les termes de l'historien d’art Benjamin Buchloh : «La rhétorique de l'exposition nazie était incitative et prohibitive ; elle prétendait avoir rétabli le critère normatif selon lequel les productions artistiques devraient désormais être conçues, produites et présentées.» (Buchloh, 2014 p. 55)

Ce type de censure étatique n'a pas seulement visé les arts visuels. Une plus petite exposition de «Entartete Musik» a rapidement suivi, mentionnant des compositeurs juifs ou des compositeurs ayant développé un style plus moderne et avant-gardiste dans leurs travaux. Dans le même état d'esprit, le jazz (qui a ses origines dans la musique des esclaves noirs) et la musique folklorique de l'Europe de l'Est étaient également considéres comme non grata, car étroitement liés à des identités particulières non germaniques et pouvaient par conséquentévoquer des sentiments nationalistes contre la suprématie allemande. De nombreux compositeurs, tels Arnold Schônberg, Kurt Weill ou Erich Wolfgang Korngold ont fuile pays pour sinstaller aux Etats-Unis. Dautres ont décidé de rester, souvent avec des conséquences tragiques, comme cela fut le cas pour le compositeur Pavel Haas. Celui-ci fut en effet déporté à Theresienstadt en 1941, dernière étape avant Auschwitz, où il mourut dans les chambres à gaz en 1944 
Theresienstadt, une ville modèle en Bohème

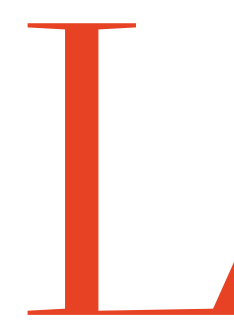
tant pour la musique et la culture parmi les nombreux camps et ghetto en Europe fut Theresienstadt (nom allemand pour Terezín). C'est l'un des lieux abordés par Amaury du Close dans son article. De nombreux vieillard et intellectuels juifs y furent emprisonnés, dont ungrand nombre de musicien et de compositeurs. Il existe quantite de récits de survivants, qui nous permettent d'avoir un aperçu de la double nature de cet endroit unique. Certaine personnes semblent ne pas avoir eu connaissance de ce qui se passait à l'extérieur, passant leurs journées en compagnie de la musique, malgré de rudes conditions de vie. D autres ont probablement su ce qui les attendait, mais se raccrochaient dans une sorte despoir ultime aux événements culturels de la ville. Pendant une courte période, Theresienstadt est presque devenue lieu idyllique que les Allemands prétendaient. Pour repondre aux rumeurs e aux critiques internationales concernant la destruction massive du peupl juif, la Croix-Rouge international fut invitee à s'y rendre pour inspecter le "ghetto modèle». La ville fut alor nettoyée, les bâtiments repeints et les habitants correctement habillés. Un représentation du célèbre opéra populaire Brundibár, créé à Theresienstad par le compositeur juif tchèque Han Krása, fut utilisée pour montrer lajoie et e bonheur des musiciens et des spectateurs. Cetévénement constitue l'un des exemples de propagande nazie les plus frappants, y compris pour l'usage délibéré de la musique. Un film fut tourné, dans le même but, et distribué sous le titre Theresienstadt. Ein Dokumentarfilm aus dem jüdischen Siedlungsgebiet. Le film était également connu sous le titre Der Führer schenkt den Juden eine Stadt. En réalité, Theresienstadt était un lieu épouvantable, comme les nombreux autres camps ou ghettos. Prè de 34000 personnes y sont mortes, et 88000 personnes furent déportées ver des camps dextermination, principalement à Auschwitz.

Parmi ces déportés se trouvait Viktor Ullmann, fils d'un officier juif autrichien. Ullmann avait fait ses étude avec Arnold Schönberg, compositeu (juif) dont la musique était clairement entartet en raison de son langage atonal et moderne. Ensemble, ils on fondé la «Verein für Musikalische Privataufführungen », une société qui a permis et promu les performances de compositions modernes au début de années 1920. Lorsque le parti national-socialiste s'est emparé du pouvoi en Allemagne, Ullmann vivait à Stuttgart, qu'il a immédiatement quittée pour Prague. Le 8 septembre 1942, il fut déporte a Terezín, où son talent musical fut reconnu. Il y joua un rôle important dans la vie musicale, mais le 16 octobre 1944, il fut déporté Auschwitz ou il mourut deux jours plus tard dans une chambre à gaz. I n’assistera donc jamais à la première de son opéra Der Kaiser von Atlantis. Il n’y aura même aucune représen- tation jusqu'en 1975. Nombre de ses autres compositions créées à Terezí ont survécu, l'une d'elles étant la pièce maîtresse du dernier disque d'Hélio Azoulay et l'Ensemble de Musique Incidentale. Avant d'être transféré Auschwitz, Ullmann travaillait su un autre opéra racontant l'histoire de Jeanne d'Arc. Le livret a été préserv dans sa totalité (et est offert dans ce dossier, ainsi que le disque), mais il ne subsiste que deux pages de musique. Hélios Azoulay et les musiciens on pris ce fragment comme point de depar d'une nouvelle aventure musicale. L'entretien réalisé par Daniel Weyssow de l Fondation Auschwitz montre que mêm aujourd hui,la créativitétrouve son orgine dans ces événements historiques.

\section{La musique dans les camps de la mort}

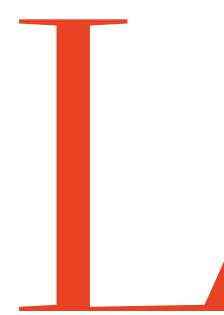

e dernier endroit où l'on s'attendrait à entendre de musique est un centre d'extermination. Dans sa contribution au présent dossie Bruno Giner met laccent sur les pratiques dans les camps de lAktion Reinhardt, operation expéditive du régime nazi pour «résoudre» la question juive. Il décrit les premiers exemples de propagande musicale et donne un aperçu détaillé des situations spécifiques $\mathrm{d}$
Bełżec, Sobibór et Treblinka. La vie schizophrénique, incluant cérémonies enarage et massacres dans les mêmes camps, est illustrée par l'ajout de plusieurs chants de camp typiques. Ces paroles ont dû résonner maintes fois dans l'enceinte des barbelés, chantées par des détenus jeunes ou vieux. Pour conclure, Bruno Giner se demande si l'usage trompeur de la musique dans cet environnement devrait nous inciter àla hair. Si la musique peut servir des objectifs bien au-delà de la dignité humaine, pouvons-nous continuer à la chérir en tant que l'une des formes d'art les plus élevées?

Dans son étude sur la musique dans les camps de concentration entre 1933 et 1945, Guido Fackler s'intéresse à la façon dont la musique faisait partie du quotidien des détenus, à Dachau, à Auschwitz, à Mauthausen, et dans de nombreux autres camps. Sa recherche se base sur letude d'archives (notamment des récits de souvenirs et un large corpus de litterature secondaire) et, dans une large mesure, sur des entretiens avec des survivants. Guido Fackler effectue une distinction entre la musique jouée ou creée par ordre des SS et celle qui émane del initiative des prisonniers. La première catégorie couvre plusieurs situations de routine: chants collectifs lors de déplacements, chants humiliants lors dexecutions ou duran le travail. La musique provenait également de haut-parleurs, initialement pour reéduquer les détenus. Associée à la diffusion des actualites relatives aux victoires d'Hitler, la musique de marches militaires allemandes jouée a dû avoir un énorme effet démoralisant.

Outre l'usage disciplinaire de la musique, Fackler souligne les repré- 
sentations des détenus pendant des fêtes privées ou officielles, ou lors de rassemblements d'officiers SS. Dans u tel contexte, la musique populaire qu aurait été considérée comme entartet par le régime officiel était également jouée, et selon Fackler, les membres SS sejoignaient même occasionnellemen aux prisonniers pour contribuer a spectacle. Cela démontre qu’à l'intérieur même des limites des camps, de sociétés miniatures se sont développées, dans lesquelles les hiérarchies les règles pouvaient parfois déroger à la politique nazie.

L'aspect le plus remarquable de la musique dans les camps est peut-êtr l'existence de tant d'ensembles muscaux organises, allant de petits groupes de deux ou trois personnes jusquà de grands orchestres symphoniques et des chœurs. Fackler fournit un aperçu general des aspects les plus importan de ce phénomène particulier, qui fa l'objet dun examen et d'un développement approfondis dans la contribution d'Amaury du Closel.

\section{Identité}

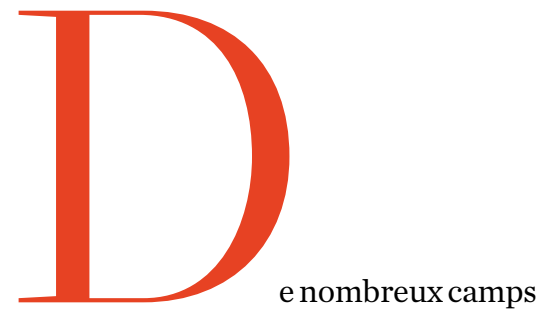

comportaient un système selon leque une autorité était accordée à certains prisonniers pour l'exercer sur d'autres, aboutissant à une forme très restreinte d'autonomie gouvernementale. De ce fait, les performances musicales pouvaient aussi provenir d'initiative spontanees, destinées a reconforte es détenus ou à leur rappeler leur vie d'avant. Dans un monde ou chaque type d'identité est ôté en rasant la tête des personnes et en tatouant un numéro su leur corps dénudé, la musique, et plus particulièrement la musique ayant un caractère folklorique, peut être un outil puissant pour établir un sentiment de communauté. Cette notion didentite est centrale dans le texte de Minako Waseda sur le rôle de la musique dans le camps de Japonais-Américains installés après l'attaque de Pearl Harbor par le Japon. Plus de 120000 descendants de Japonais, la plupart nés aux États-Unis, se sont vus dépouillés de tout et envoyés dans des camps improvisés basés dan des zones eloignees. Contrairement aux camps allemands, ils n’étaient pas destinés a exterminerlapopulationjaponaise des États-Unis. L'objectif principal étai d'écarter de la société toute personne ayant du sang japonais pour eviter de emeutes anti-americaines en cas d'invasion nippone. La politique officielle de la War Relocation Authority (autorité de relogement par temps de guerre) étai deles «américaniser». Minako Waseda decrit comment la vie dans ces camp était, d'une certaine façon, culturellement riche, en raison de la concentration importante de maitres dans le arts traditionnels japonais et de l'abondance de temps de loisirs accordés par les autorites pour maintenir une paix et une tranquillité relatives. Cet épisode particulier de l'histoire americaine est longtemps resté sous-exposé, jusquaà la diffusion du film documentaire Hidden Legacy (2014), qui l'a révélé dans toute sa complexité et a (re)formé la mémoire collective.

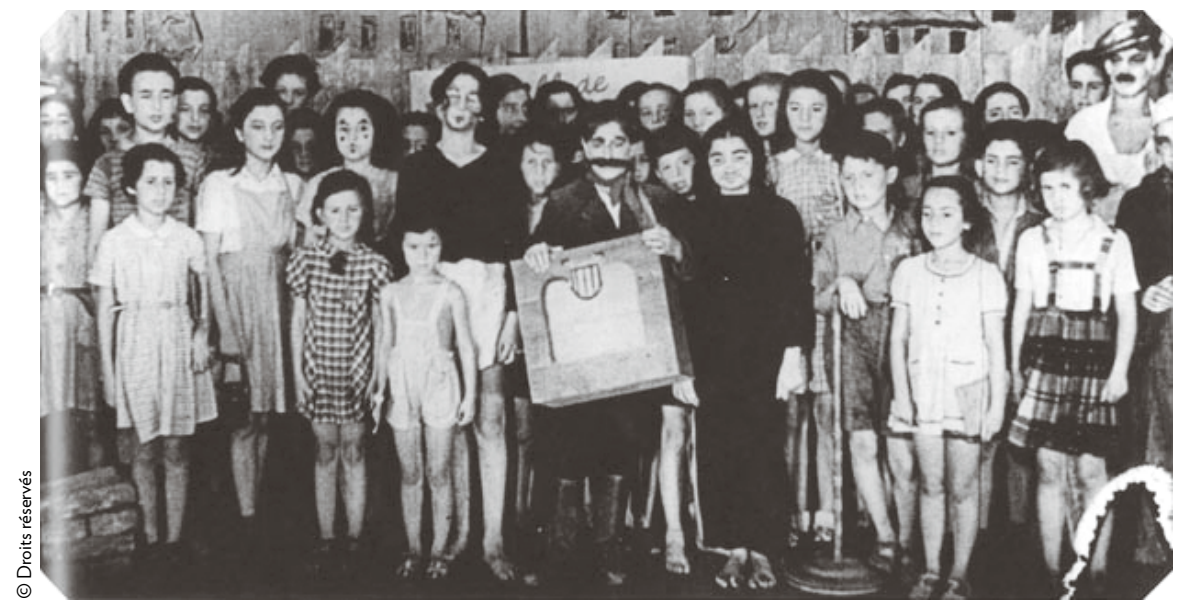

- Photo de propagande prise par les nazis lors d'une représentation de
Brundibár à Theresienstad

Ce dossier montre à quel point $\mathrm{l}$ musique était un aspect important de la vie dans les camps et les ghettos de années 1,30et 1940, à la fois en Europe et aux Etats-Unis. Elle était utilisée pour la propagande, le divertissement. à des fins militaires, et parfois comm réconfort. C'était un moyen de resserrer les liens avec les autres détenus e de recouvrer une identite perdue. Dan certains cas, la musique etait une bouée de sauvetage. Danslefilm documentaire The Lady in Number 6: Music Saved My Life (2013), Anita Lasker-Wallfish, violoncelliste et survivante de la Shoah, se rappelle le conseil de son père : «Mets tout ce que tu peux dans ta tête, car c'es quelque chose que personne ne peut t'enlever.»

Klaas Coulembier KU Leuve Traduit de l'anglais par Carine Chaura
DUVRAGES CITÉs

- Buchloh, Benjamin (2015) Destruction: The Degenerate Art Exhibition (1937) and the Surréalisme (1938)', October 150 - Deleuze, Gilles \& Félix Guattar (1980): Mille Plateaux, P -..., A Thousand Plateaus,
traduit du francais par Brian (radui dh trançais par Brian

- Schuster, Peter-Klaus (dir.) 'Entartete Kunst'. Die Kunststadt' München 1937, München, Prestel- 